\title{
Baseline higher peritoneal transport had been associated with worse nutritional status of incident continuous ambulatory peritoneal dialysis patients in Southern China: a 1-year prospective study
}

\author{
Yun Liu ${ }^{1,2,3} \dagger$, Rong Huang ${ }^{1,2,3} \dagger$, Qunying Guo ${ }^{1,2,3}$, Qiongqiong Yang ${ }^{1,2,3}$, Chunyan $\mathrm{Yi}^{1,2,3}$, \\ Jianxiong Lin ${ }^{1,2,3}$, Xueqing $\mathrm{Yu}^{1,2,3}$ and Xiao Yang ${ }^{1,2,3 *}$ \\ ${ }^{1}$ Department of Nephrology, The First Affiliated Hospital, Sun Yat-sen University, 58th Zhongshan Road II, \\ Guangzhou 510080, China \\ ${ }^{2}$ Key Laboratory of Nephrology, Ministry of Health, Guangzhou 510080, China \\ ${ }^{3}$ Guangdong Provincial Key Laboratory of Nephrology, Guangzhou 510080, China \\ (Submitted 19 October 2014 - Final revision received 16 February 2015 - Accepted 27 April 2015 - First published online 16 July 2015)
}

\section{Abstract}

The aim of the present study was to investigate the relationship between baseline peritoneal transport types and nutritional status in Chinese continuous ambulatory peritoneal dialysis (CAPD) patients. In the present single-centre, prospective study, incident CAPD patients were included from 15 April 2010 to 31 December 2011 and were followed up for 12 months. According to the results of baseline peritoneal equilibration test, patients were divided into lower peritoneal transport group (lower transporters) and higher peritoneal transport group (higher transporters). Nutritional status was evaluated by both subjective global assessment (SGA) and protein-energy wasting (PEW) score. The body composition parameters were assessed by body impedance analysis. A total of 283 CAPD patients were included in the study, of which $171(60 \cdot 4 \%)$ were males with a mean age of 47.0 (sD 14.9) years. Compared with lower transporters ( $n$ 92), higher transporters ( $n$ 181) had lower levels of serum albumin (37.1 (SD 4.3) v. 39.6 (SD 4.3) g/l, $P<0.001$ ), serum pre-albumin (356 (SD 99$) v .384$ (SD 90$) \mathrm{mg} / \mathrm{l}, P=0.035$ ), phase angle $(6.15 \text { (SD 0.39) } v .6 .27 \text { (SD } 0.47)^{\circ}, P<0.05$ ) and higher rate of malnutrition defined by SGA $(52.5 v$. $25.0 \%, P<0.001)$ and PEW score $(37.0 \mathrm{v} .14 .1 \%, P<0.001)$ at 1 -year of follow-up. Baseline higher peritoneal transport, analysed by multivariate binary logistic regressions, was independently associated with malnutrition (SGA mild to moderate and severe malnutrition: OR 3.43, $95 \%$ CI 1.69, 6.96, $P<0 \cdot 01$; PEW: OR 2.40, $95 \%$ CI 1·08, 5.31, $P=0 \cdot 03$ ). It was concluded that baseline higher peritoneal transport was independently associated with worse nutritional status of CAPD patients in Southern China.

Key words: Continuous ambulatory peritoneal dialysis: Peritoneal equilibrium test: Peritoneal transport: Malnutrition

Malnutrition has been associated with increased morbidity and mortality in patients undergoing continuous ambulatory peri toneal dialysis $(\mathrm{CAPD})^{(1)}$. Although much progress has been made on improving the nutritional status of CAPD patients, the prevalence of protein-energy malnutrition is still high (ranging from 18 to approximately $56 \%$, depending on the assessment methods used $)^{(1)}$. The anorexia due to abdominal distension and glucose absorption from the dialysate, loss of nutrients in dialysate, inflammation and loss of residual renal function (RRF) could be responsible for malnutrition in CAPD patients.
Individual differences in peritoneal membrane function have been shown to influence the clinical outcomes in patients undergoing $\mathrm{CAPD}^{(2)}$. The higher peritoneal transport is a status of a high peritoneal small-solute transport rate, which includes both peritoneal high average and high transport status determined by peritoneal equilibration test $(\mathrm{PET})^{(3)}$. The protein-energy wasting (PEW) reflects malnutrition and wasting caused not only by inadequate nutrient intake but also by depletion resulting from the inflammatory and non-inflammatory conditions that prevail in dialysis population $^{(4)}$. It had been reported that higher peritoneal transport

Abbreviations: $4 \mathrm{~h}$ D:P cr, creatinine ratio of dialysate:plasma at $4 \mathrm{~h}$ of the peritoneal equilibrium test; ALB, albumin; CAPD, continuous ambulatory peritoneal dialysis; hsCRP, high-sensitive C-response protein; mBIA, multiple-frequency bioelectrical impedance analysis; PA, phase angle; PD, peritoneal dialysis; PET, peritoneal equilibration test; PEW, protein-energy wasting; pre-ALB, pre-albumin; RRF, residual renal function; SGA, subjective global assessment.

* Corresponding author: Dr X. Yang, fax +8620 87769673, email yangxsysu@126.com

†Yun Liu and Rong Huang contributed equally to this study. 
status is associated with high baseline albumin clearance ${ }^{(5)}$, and recently, the Global Fluid Study investigators demonstrated that higher transport status was linked with greater levels of intraperitoneal inflammation ${ }^{(6)}$. Therefore, it had been suggested that higher peritoneal transport status might result in progressive deterioration of nutritional status in peritoneal dialysis (PD) patients. However, studies on the association between peritoneal transport and nutritional status have presented contradictory results ${ }^{(7-10)}$. In the present study, we aimed to investigate the relationship between baseline peritoneal transport types and nutritional status at the first year of CAPD in patients of Southern China.

\section{Subjects and methods}

\section{Study population}

Incident CAPD patients were enrolled in this 1-year prospective study from 15 April 2010 to 31 December 2011 at the PD centre of The First Affiliated Hospital, Sun Yat-sen University, Guangzhou, China. The inclusion criteria were as follows: (1) CAPD therapy for more than $90 \mathrm{~d}$; (2) age $\geq 18$ years; (3) use of glucose dialysate (Baxter Healthcare); (4) signed informed consent form. The exclusion criteria were as follows: patients who had episodes of peritonitis within 3 months before PET, or expected to have kidney transplantation within 6 months; or with serious liver disease; or with amputation, or with pacemaker; or with active comorbidities or recent systemic infections; or were not able to accomplish the measurement of body composition in standing position for $3 \mathrm{~min}$. All patients were followed up for 12 months. Icodextrin dialysate was not available in these patients. The study protocols were approved by the Ethics Committee of The First Affiliated Hospital of Sun Yat-sen University.

The following demographic and clinical variables were collected at the start of CAPD: age; sex; body weight and height; primary renal disease; modified Charlson comorbidity index ${ }^{(11)}$; clinical symptom; blood pressure; medical prescription; CAPD regimen; $24 \mathrm{~h}$ urine output; total peritoneal ultrafiltration; night dwell peritoneal ultrafiltration. Data on serum albumin (ALB), pre-albumin (pre-ALB), transferrin, $\mathrm{Hb}$, high-sensitive C-response protein (hsCRP), total cholesterol and $\mathrm{Kt} / \mathrm{V}$ were obtained at the baseline (0 month), 6 months and 12 months of follow-up, respectively. Prescription of each CAPD patient was recorded by the primary nurses and was used for estimating peritoneal glucose absorption, which was expressed as averaged glucose dose per d ( glucosedose(g)/vintage(d)), where $\Sigma$ is the total glucose dose used during the follow-up period. The weekly total $\mathrm{Kt} / \mathrm{V}$ (urea clearance index used to quantify the dialysis treatment adequacy, where $\mathrm{K}$ is the dialyser clearance of urea, $t$ is the dialysis time and $\mathrm{V}$ is the volume of distribution of urea approximately equal to total body water of the patient) was measured by the methods described by Lysaght et $a l^{(12)}$. Residual kidney function was measured by mean values of creatinine clearance and urea clearance and adjusted for body surface area. All patients were followed up for 12 months. The causes and days for hospitalisation, change of medical and PD prescription, and reasons for withdrawal of PD during the follow-up period were also recorded.

\section{Peritoneal transport status}

A $4 \mathrm{~h}$ PET, as described by Twardowski et al. ${ }^{(13)}$, was performed on each patient within the first 1-3 months after CAPD. Dialysate samples were taken at 0,2 and $4 \mathrm{~h}$ of dwell while blood samples were obtained at $2 \mathrm{~h}$. Ratios of D:P creatinine were calculated using dialysate (D) creatinine concentrations at $4 \mathrm{~h}$ of PET divided by the plasma (P) creatinine concentrations ( $4 \mathrm{~h}$ D:P cr). Peritoneal transport status was considered a categorical variable according to the four groupings of $4 \mathrm{~h}$ D:P cr values defined by Twardowski et al. ${ }^{(13)}$ : low $<0.50$; low average 0.50-0.64; high average 0.65-0.80; high $>0 \cdot 81$. Patients were divided into lower peritoneal transport group (including low, low average transporters) and higher peritoneal transport group (including high average, high peritoneal transporters).

\section{Measurement of nutritional status}

Subjective global assessment. At the baseline and 12 months of follow-up, subjective global assessment (SGA) score was calculated using the method described by Detsky et al. ${ }^{(14)}$, which was widely applied in CAPD patients ${ }^{(15,16)}$. According to the score, nutritional status was classified as severe malnutrition ( $\mathrm{SGA}=1-2, \mathrm{C}$ ), mild-to-moderate malnutrition $(\mathrm{SGA}=3-5, \mathrm{~B})$ and good nutritional status (SGA = 6-7 A). In the present study, patients were grouped into wellnourished (SGA A) and malnourished (SGA B + C) categories for the purpose of statistical analysis.

Bioimpedance analysis. Multiple-frequency bioelectrical impedance analysis (mBIA; InBody 720) was conducted on the day of PET in fasting condition in the morning, the analysis being conducted three times to get rid of the bias. Phase angle (PA) was applied as the main nutritional parameter, which was calculated directly from reactance and resistance. PA was calculated as arc-tangent reactance/resistance $\times 180^{\circ} / \pi$, with a frequency of $50 \mathrm{kHz}^{(17)}$. Extracellular water, intracellular water, lean body mass, skeletal muscle mass and fat mass percentage were also measured indirectly by mBIA. Data on mBIA were collected at the baseline, 6 months and 12 months of the follow-up.

Protein-energy wasting score. The diagnostic criteria proposed by the International Society of Renal Nutrition and Metabolism panel ${ }^{(1,18)}$ were taken into reference; however, some criteria were adjusted for Asian population. The diagnostic criteria of PEW in the present study were as follows: (1) serum chemistry: serum ALB level $<38 \mathrm{~g} / \mathrm{l}$, serum preALB level $<300 \mathrm{mg} / \mathrm{l}$, serum cholesterol level $<2.59 \mathrm{mmol} / \mathrm{l}$; (2) body mass: BMI $<18.5 \mathrm{~kg} / \mathrm{m}^{2(19)}$, unintentional non-oedematous weight loss over time $\geq 5 \%$ over 3 months or $\geq 10 \%$ over 6 months (non-oedematous weight = body weight excessive water measured by mBIA), total body fat percentage $<10 \%$ (measured by mBIA); (3) muscle wasting: reduced 
muscle mass $\geq 5 \%$ over 3 months or $\geq 10 \%$ over 6 months (measured by mBIA); (4) protein metabolism: normalised protein catabolism rate $<1 \mathrm{~g} / \mathrm{kg}$ per $\mathrm{d}$ for at least 2 months. A diagnosis of PEW requires that at least three of the four listed categories should be met and at least one test in each of the selected category should be included. PEW was assessed at 12 months of follow-up. During management of the patients, when serum ALB levels of the patients were less than $38 \mathrm{~g} / \mathrm{l}$, keto acids (Fresenius Kabi) were prescripted for these patients routinely, and the dosage range was three to four tablets three times per $\mathrm{d}$ according to body weight.

\section{Statistical analysis}

Data were presented as means and standard deviations or medians and interquartile range for continuous variables and number (percentages) for categorical variables. Differences between the two groups were evaluated by Student's $t$ test or Mann-Whitney test or the $\chi^{2}$ test as appropriate. Serial changes of nutritional parameters were compared with the baseline values according to patient group using ANOVA for repeated measures. Multivariate linear regressions with stepwise, both univariate and multivariate binary logistic regression analysis with forward conditional methods, were performed to determine the variables that significantly affect nutritional status. A $P$ value $<0.05$ was considered statistically significant. SPSS version 16.0 for Windows (SPSS, Inc.) was used for the statistical analysis.

\section{Results}

\section{Demographic and clinical characteristics of study population}

A total of 283 CAPD patients with $171(60 \cdot 4 \%)$ male and mean age 47.0 (SD 14.9) years were recruited (Fig. 1). The median PD duration at the time of recruitment was 45 (range $41-72) \mathrm{d}$. The primary cause of end-stage renal disease was chronic glomerulonephritis ( $n$ 162; 57.2\%), followed by diabetic nephropathy ( $n$ 58; 20.5\%) and hypertension ( $n$ 17; $6.0 \%$ ). The average $4 \mathrm{~h} \mathrm{D:P}$ cr of all patients at baseline was 0.69 (SD 0.16.). Of the total patients, fourteen (4.9\%) were low transporters, $79(27.9 \%)$ were low average transporters, $140(49 \cdot 5 \%)$ were high average transporters and $50(17 \cdot 7 \%)$ were high transporters. Compared with lower transporters,

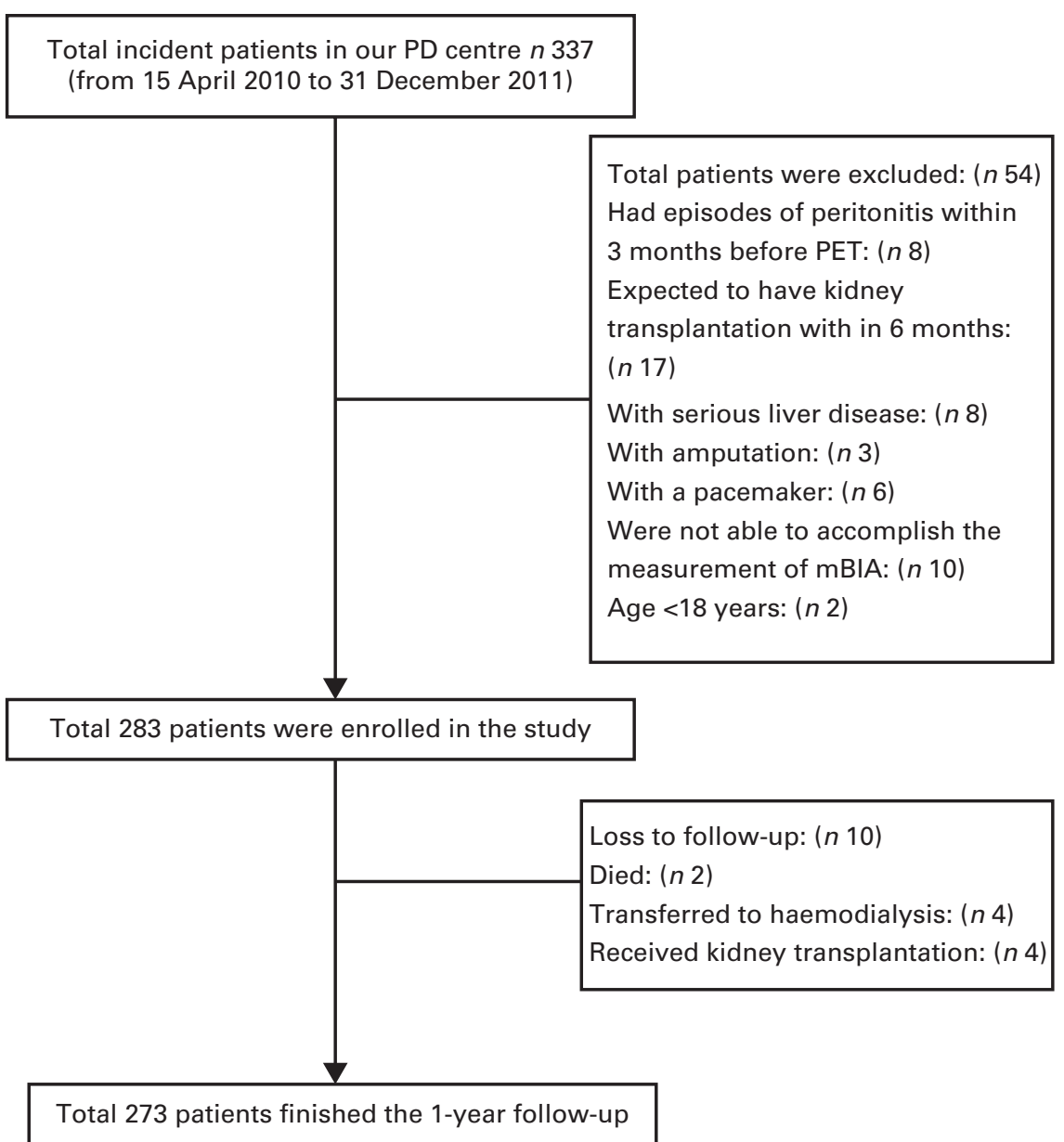

Fig. 1. Derivation of the study sample. PD, peritoneal dialysis; PET, peritoneal equilibration test; mBIA, multiple-frequency bioelectrical impedance analysis. 
Table 1. Demographic and clinical characteristics of the study population at baseline

(Mean values, standard deviations, number of subjects, percentages and ranges)

\begin{tabular}{|c|c|c|c|c|c|}
\hline \multirow[b]{2}{*}{ Variables } & \multicolumn{2}{|c|}{ Lower transporters ( $n$ 93) } & \multicolumn{2}{|c|}{ Higher transporters ( $n$ 190) } & \multirow[b]{2}{*}{$P$} \\
\hline & Mean & SD & Mean & SD & \\
\hline Age (years) & $46 \cdot 5$ & $13 \cdot 7$ & $47 \cdot 3$ & $15 \cdot 5$ & 0.65 \\
\hline Sex (male) & & & & & $<0.01$ \\
\hline$n$ & \multicolumn{2}{|c|}{47} & \multicolumn{2}{|c|}{124} & \\
\hline$\%$ & \multicolumn{2}{|c|}{$50 \cdot 5$} & \multicolumn{2}{|c|}{$65 \cdot 2$} & \\
\hline Diabetes mellitus & \multirow{2}{*}{\multicolumn{2}{|c|}{17}} & & & $<0.01$ \\
\hline$n$ & & & \multirow{2}{*}{\multicolumn{2}{|c|}{44}} & \\
\hline$\%$ & \multicolumn{2}{|c|}{$18 \cdot 3$} & & & \\
\hline Body weight (kg, oedema-free) & $55 \cdot 8$ & $16 \cdot 2$ & $54 \cdot 2$ & $17 \cdot 4$ & 0.46 \\
\hline BMI $\left(\mathrm{kg} / \mathrm{m}^{2}\right)$ & $22 \cdot 3$ & 3.5 & 22.5 & $3 \cdot 3$ & 0.61 \\
\hline Modified CCI & \multicolumn{2}{|c|}{$\begin{array}{c}0 \\
0-2\end{array}$} & \multirow{2}{*}{\multicolumn{2}{|c|}{$\begin{array}{c}1 \\
0-2\end{array}$}} & 0.02 \\
\hline Range & & & & & \\
\hline $4 \mathrm{~h} \mathrm{D}: \mathrm{P}$ cr & 0.59 & 0.02 & 0.76 & 0.09 & $<0.01$ \\
\hline
\end{tabular}

$\mathrm{CCl}$, Charlson comorbidity index; $4 \mathrm{~h} \mathrm{D:P} \mathrm{cr}$, creatinine ratio of dialysate:plasma at $4 \mathrm{~h}$ of the peritoneal equilibrium test.

higher transporters were predominantly male, had higher proportion of diabetes mellitus and higher Charlson comorbidity index (Table 1). At the initiation of CAPD, patients with higher transport had higher systolic blood pressure, serum hsCRP, serum creatinine, poor appetite, as well as lower total peritoneal ultrafiltration, night dwell peritoneal ultrafiltration and fat mass percentage (Table 2).

At 12 months of follow-up, ten (3.5\%) patients dropped out, among whom two patients with higher transport died, four patients transferred to haemodialysis (one lower transporters and three higher transporters) and four patients with higher transport received kidney transplantation (Fig. 1). Higher transporters had higher rate of poor appetite, lower total peritoneal ultrafiltration, night dwell peritoneal ultrafiltration, fat mass percentage as well as higher peritoneal glucose absorption per $\mathrm{d}$, and higher episodes of peritonitis. There were no significant difference in RRF, weekly total $\mathrm{Kt} / \mathrm{V}$ urea, $\mathrm{Hb}$ and serum transferrin between the two groups both at the baseline or 12 months of follow-up (Table 2).

\section{Nutritional status between the two groups at 0 and 12 months}

At the initiation of $\mathrm{CAPD}$, the total malnourished patients defined by SGA B +C were 56.5\%. Compared with lower transporters, higher transporters had lower serum ALB, serum pre-ALB, PA and were more frequent with malnourished conditions defined by SGA (62.6 v. 45.2\%; Table 2; Fig. 2).

At 12 months of follow-up, the total malnourished patients defined by SGA B $+\mathrm{C}$ were $43 \cdot 2 \%$ and by PEW score were $29.3 \%$. Patients in both groups had increased level of serum ALB. However, the rate of malnourished conditions defined by SGA and PEW score were significantly higher (52.5 $v$. $25.0 \%, P<0.001 ; 37.0$ v. 14.1\%, $P<0.001)$, and the levels of serum ALB (37.1 (sD 4.3) v. 39.6 (sD 4.3) g/l, $P<0 \cdot 001$ ), serum pre-ALB (356 (sD 99) v. $384(\mathrm{sD} 90) \mathrm{mg} / \mathrm{l}, P=0.035)$, PA $\left(6.15(\mathrm{SD} 0.39) \quad v .6 .27(\mathrm{SD} 0.47)^{\circ}, \quad P<0.05\right)$, skeletal muscle mass (25.2 (SD 4.3) v. 26.9 (SD 5.1) kg, $P<0.05)$ were lower than that of lower transporters (Table 2; Fig. 2).

\section{The associations of higher transport status and malnutrition of patients at 12 months of follow-up}

The associations of baseline peritoneal transport status and malnutrition of patients at 12 months of follow-up with defined binary logistic regression models are listed in Table 3. After adjustment of ageing (age $>65$ years), sex, diabetes mellitus, RRF, Kt/V, peritonitis incidence and serum hsCRP, as well as keto acid supplementation, baseline higher transport status was independently associated with malnutrition defined by SGA score (OR 3.43, 95\% CI 1.69, 6.96, $P<0 \cdot 01)$. Similarly, baseline higher transport status was independently associated with malnutrition defined by PEW score (OR 2.40, 95\% CI 1.08, 5.31, P<0.01). Furthermore, after adjusting the same variables as mentioned above, multiple linear regression results showed that baseline higher transporter was independently associated with lower serum $\operatorname{ALB}(\beta=-0.164, P=0.004)$ and PA $(\beta=-0.125, P=0.009)$ measured by mBIA.

\section{Hospitalisation}

There were seventy-four hospitalisations among 283 patients during the follow-up period ( $0 \cdot 261$ time/patient year). Primary causes for hospitalisation included peritonitis (twenty-seven hospitalisations, 0.095 time/patient year), inadequate dialysis (nineteen hospitalisations, 0.067 time/patient year) and nonperitonitis infection (eight hospitalisations, 0.028 time/patient year). Patients with higher peritoneal transporter status had significantly higher total rate of hospitalisation $(0.32(0-3) v$. $0 \cdot 13(0-2)$ time/patient year, $P<0 \cdot 01)$ and the hospitalisation rate due to peritonitis $(0 \cdot 14(0-3) v \cdot 0 \cdot 01(0-1)$ time/patient year, $P<0.01)$. Because only two patients died, the multivariate Cox regression was not analysed.

\section{Discussion}

The present prospective study showed that both at the baseline or 12 months of follow-up, higher transporters had higher rate of malnutrition than that of lower transporters, 
Table 2. Clinical parameters of higher transporter and lower transporter at 0 and 12 months

(Mean values, standard deviations, medians, interquartile ranges, number of subjects and percentages)

\begin{tabular}{|c|c|c|c|c|c|c|c|c|c|c|}
\hline & \multicolumn{5}{|c|}{0 months ( $n$ 283) } & \multicolumn{5}{|c|}{12 months ( $n$ 273) } \\
\hline & \multicolumn{2}{|c|}{ Lower transporter (n 93) } & \multicolumn{2}{|c|}{ Higher transporter $(n$ 190) } & \multirow[b]{2}{*}{$P$} & \multicolumn{2}{|c|}{ Lower transporter ( $n$ 92) } & \multicolumn{2}{|c|}{ Higher transporter ( $n$ 181) } & \multirow[b]{2}{*}{$P$} \\
\hline & Median & Interquartile range & Median & Interquartile range & & Median & Interquartile range & Median & Interquartile range & \\
\hline \multicolumn{11}{|l|}{ Overall nutritional status } \\
\hline SGA B $+C^{*}$ & & & & & $<0.01$ & & & & & $<0.01$ \\
\hline$n$ & & 42 & & 119 & & & 23 & & 95 & \\
\hline$\%$ & & $45 \cdot 2$ & & $62 \cdot 6$ & & & $25 \cdot 0$ & & $52 \cdot 5$ & \\
\hline PEW & & & & & & & & & & $<0.01$ \\
\hline$n$ & & & & & & & 13 & & 67 & \\
\hline$\%$ & & & & & & & $14 \cdot 1$ & & $37 \cdot 0$ & \\
\hline \multicolumn{11}{|l|}{ Biochemical data } \\
\hline Serum hsCRP (mg/l) & 1.69 & $0.83-3.23$ & $2 \cdot 23$ & $0.66-7.59$ & 0.04 & 2.06 & $1.88-8.73$ & 1.49 & $0.60-5.35$ & 0.11 \\
\hline Serum $\mathrm{Hb}(\mathrm{g} / \mathrm{l})$ & & & & & 0.25 & & & & & 0.60 \\
\hline Mean & & 107 & & 103 & & & 110 & & 109 & \\
\hline SD & & 19 & & 22 & & & 20 & & 20 & \\
\hline Serum total cholesterol $(\mathrm{mmol} / \mathrm{l})$ & 4.91 & $4.22-5.91$ & 5.03 & $4 \cdot 22-5 \cdot 70$ & 0.36 & 4.80 & $3.80-5.70$ & 5.00 & $3.93-5.71$ & 0.71 \\
\hline Serum transferrin $(\mathrm{g} / \mathrm{l})$ & $2 \cdot 16$ & $1 \cdot 7-2 \cdot 45$ & 2.00 & $1 \cdot 7-2 \cdot 4$ & 0.15 & $2 \cdot 19$ & $1.87-2.52$ & $2 \cdot 11$ & $1.79-2.52$ & 0.64 \\
\hline Serum urea $(\mathrm{mmol} / \mathrm{l})$ & $17 \cdot 1$ & $13 \cdot 2-22 \cdot 0$ & $15 \cdot 7$ & $12 \cdot 6-19 \cdot 9$ & 0.27 & $18 \cdot 0$ & $14 \cdot 8-22 \cdot 1$ & $17 \cdot 8$ & $15 \cdot 1-21 \cdot 9$ & 0.57 \\
\hline Serum creatinine $(\mu \mathrm{mol} / \mathrm{l})$ & 725 & $571-894$ & 755 & $615-918$ & 0.04 & 926 & $732-1182$ & 900 & $669-1137$ & 0.55 \\
\hline \multicolumn{11}{|l|}{ Body composition (by mBIA) } \\
\hline ECW/TBW & & & & & 0.08 & & & & & 0.78 \\
\hline Mean & & 0.39 & & 0.40 & & & 0.38 & & 0.39 & \\
\hline SD & & 0.01 & & 0.03 & & & 0.06 & & 0.06 & \\
\hline Fat mass percentage & $14 \cdot 8$ & $10 \cdot 3-22 \cdot 1$ & $20 \cdot 2$ & $13 \cdot 9-27 \cdot 4$ & $<0.01$ & $22 \cdot 5$ & $14 \cdot 5-28 \cdot 7$ & $18 \cdot 8$ & $12 \cdot 0-24 \cdot 6$ & 0.01 \\
\hline Oedema-free mass $(\mathrm{kg})$ & & & & & 0.46 & & & & & 0.74 \\
\hline Mean & & $55 \cdot 8$ & & $54 \cdot 2$ & & & $58 \cdot 6$ & & 57.9 & \\
\hline SD & & $16 \cdot 2$ & & 17.4 & & & $15 \cdot 4$ & & $16 \cdot 4$ & \\
\hline \multicolumn{11}{|l|}{ Dialysis index } \\
\hline $\operatorname{RRF}(\mathrm{ml} / \mathrm{min})$ & 3.04 & $1.88-4.31$ & $3 \cdot 13$ & $1.96-5.09$ & 0.38 & 3.03 & $1.82-4.04$ & $3 \cdot 11$ & $1.99-4.97$ & 0.48 \\
\hline Weekly total $\mathrm{Kt} / \mathrm{V}$ urea & & & & & 0.60 & & & & & 0.79 \\
\hline Mean & & $2 \cdot 41$ & & 2.45 & & & $2 \cdot 34$ & & $2 \cdot 32$ & \\
\hline SD & & 0.59 & & 0.72 & & & 0.58 & & 0.68 & \\
\hline Total peritoneal ultrafiltration (ml/d) & 522 & $300-800$ & 350 & $100-650$ & $<0.01$ & 400 & $163-744$ & 300 & $100-600$ & 0.04 \\
\hline Night dwell peritoneal ultrafiltration $(\mathrm{ml} / \mathrm{d})$ & 100 & $0-200$ & 0 & $-125-150$ & $<0.01$ & 50 & $-50-150$ & 0 & $-100-100$ & $<0.01$ \\
\hline $24 \mathrm{~h}$ urine output $(\mathrm{ml})$ & 700 & $400-1175$ & 800 & $500-1100$ & 0.39 & 800 & $500-1100$ & 700 & $300-1100$ & 0.16 \\
\hline \multicolumn{11}{|l|}{ Other clinical data } \\
\hline $\mathrm{SBP}(\mathrm{mmHg})$ & & & & & 0.02 & & & & & 0.05 \\
\hline Mean & & 128 & & 133 & & & 135 & & 140 & \\
\hline SD & & 17 & & 18 & & & 16 & & 16 & \\
\hline Decreased appetite & & & & & $<0.01$ & & & & & $<0.01$ \\
\hline$n$ & & 21 & & 81 & & & 17 & & 99 & \\
\hline$\%$ & & $22 \cdot 6$ & & $42 \cdot 6$ & & & 18.5 & & 54.7 & \\
\hline $\begin{array}{l}\text { Estimate peritoneal glucose } \\
\text { absorption }(\mathrm{kg} / 24 \mathrm{~h})\end{array}$ & & & & & & 0.12 & $0.12-0.13$ & $0 \cdot 19$ & $0.12-0.21$ & $<0.01$ \\
\hline
\end{tabular}

SGA, subjective global assessment; PEW, protein-energy wasting; hsCRP, high-sensitive C-response protein; mBIA, multiple-frequency bioelectrical impedance analysis; ECW/TBW, extracellular water/total body water; RRF, residual renal function; SBP, systolic blood pressure.

* SGA B represents a score of 3-5 (mild to moderate malnutrition); SGA C represents a score of 1-2 (severe malnutrition). 

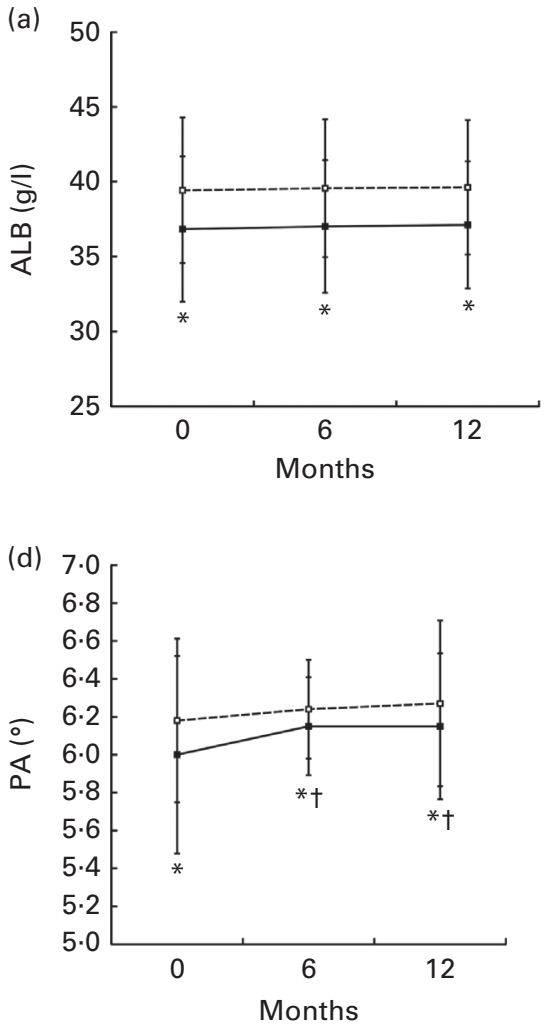
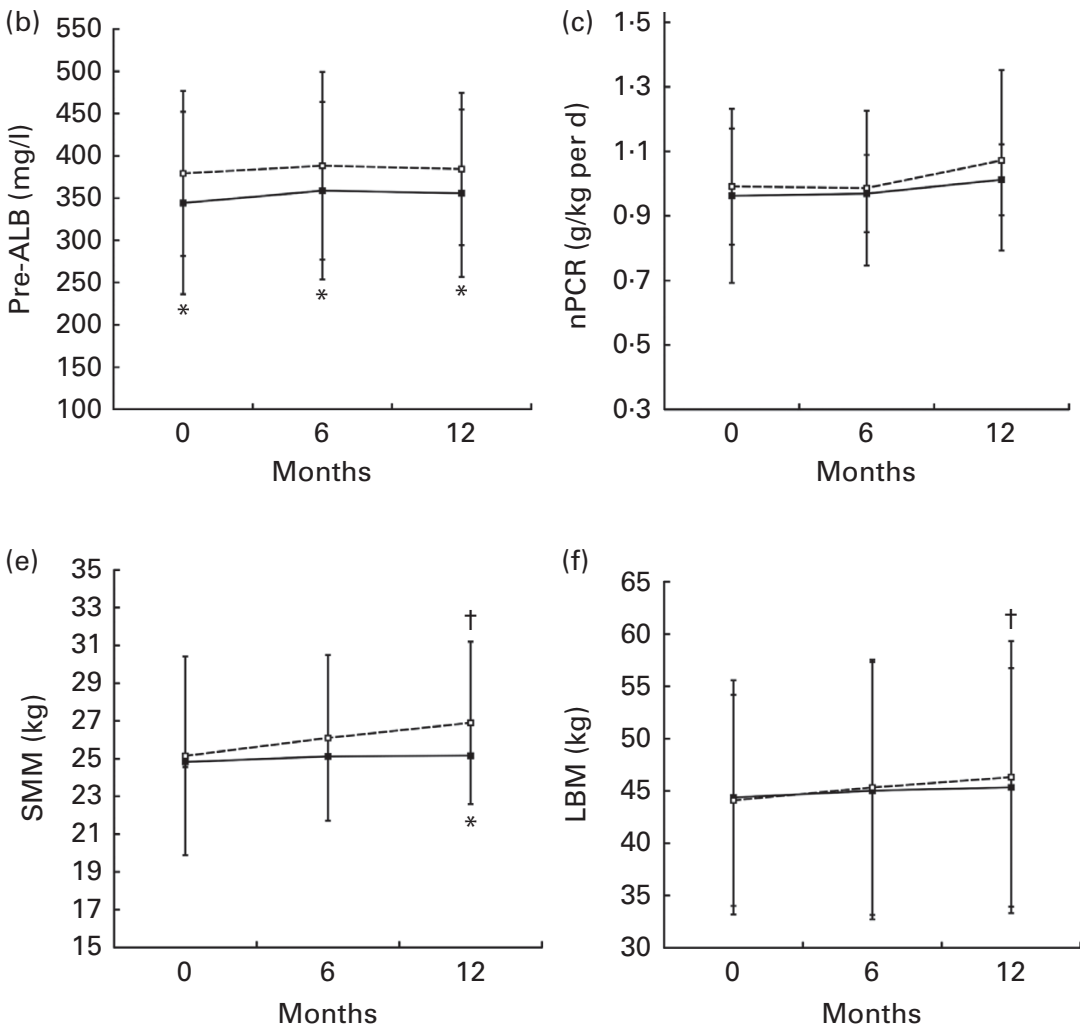

Fig. 2. Nutritional parameters of patients at 0 months, 6 months and 12 months (a) serum albumin (ALB), (b) serum pre-albumin (pre-ALB), (c) normalised protein catabolism rate (nPCR), (d) phase angle (PA), (e) skeletal muscle mass (SMM) and (f) lean body mass (LBM). * $P<0.05$, compared with lower transporter; $\dagger P<0.05$, compared with 0 month. $\square$, higher transporters; $\square$, lower transporters.

although nutritional status of the two groups was improved during the follow-up. After adjustment of age, sex, diabetes mellitus, RRF, Kt/V, peritonitis incidence and serum hsCRP, as well as keto acid supplementation, baseline higher peritoneal transport status was independently associated with poor nutritional status of patients at 12 months of follow-up.

Previous clinical studies on whether higher peritoneal transport status had been associated with overall worse nutritional status in CAPD patients have shown discrepant results. A 2-year prospective study that recruited both prevalent (177 cases) and incident (fifty-eight cases) patients by Szeto et $a l .{ }^{(9)}$ has found that peritoneal transport status had not been associated with longitudinal change of nutritional parameters assessed by serum ALB level and percentage of lean body mass. An earlier cross-sectional study (sample size: 147 CAPD patients) by Harty et $a l^{(7)}$ has found no significant relationship between $4 \mathrm{~h}$ D:P $\mathrm{cr}$ and nutritional parameters. In two cross-sectional studies with 147 and 104 prevalent CAPD patients, respectively, Kang et al. ${ }^{(8)}$ and Zhang \& Ren et $a l .{ }^{(10)}$ found that the $4 \mathrm{~h} \mathrm{D:P} \mathrm{cr}$ is an independent factor affecting the overall nutritional status measured by composite nutritional index and SGA, respectively. In our prospective study, 283 incident patients on CAPD were observed and followed up for 1 year. It was found that higher peritoneal

Table 3. Association between peritoneal transport status and subjective global assessment (SGA) $B+C^{*}$ and protein-energy wasting (PEW)

(Odds ratios and $95 \%$ confidence intervals)

\begin{tabular}{|c|c|c|c|c|c|c|}
\hline & \multicolumn{3}{|c|}{$S G A B+C$} & \multicolumn{3}{|c|}{ PEW } \\
\hline & OR & $95 \% \mathrm{Cl}$ & $P$ & OR & $95 \% \mathrm{Cl}$ & $P$ \\
\hline \multicolumn{7}{|l|}{ Unadjusted } \\
\hline $\begin{array}{l}\text { Higher transporter } v \text {. lower transporter } \\
\text { Model } 1 \dagger\end{array}$ & $3 \cdot 31$ & $1 \cdot 90,5 \cdot 77$ & $<0.01$ & 3.57 & $1 \cdot 85,6 \cdot 91$ & $<0.01$ \\
\hline $\begin{array}{l}\text { Higher transporter } v \text {. lower transporter } \\
\text { Model } 2 \ddagger\end{array}$ & 3.13 & $1 \cdot 59,6 \cdot 71$ & $<0.01$ & $2 \cdot 30$ & $1.05,5.04$ & 0.04 \\
\hline Higher transporter $v$. lower transporter & 3.43 & $1.69,6.96$ & $<0.01$ & $2 \cdot 40$ & $1 \cdot 08,5 \cdot 31$ & 0.03 \\
\hline
\end{tabular}

* SGA B represents a score of 3-5 (mild to moderate malnutrition); SGA C represents a score of 1-2 (severe malnutrition).

† Adjusted for age, sex, diabetes mellitus, residual renal function (RRF), Kt/V, peritonitis incidence and serum high-sensitive $\mathrm{C}$-response protein (hsCRP).

$\ddagger$ Adjusted for age, sex, diabetes mellitus, RRF, Kt/V, peritonitis incidence, serum hsCRP and keto acid supplementation. 
transporter was independently associated with worse nutritional status defined not only by comprehensive nutritional assessment tools such as SGA and PEW score but also by single nutritional parameters, including serum ALB level, serum pre-ALB and PA measured by bioelectrical impedance analysis, which was consistent with the results reported by Kang et $a l .{ }^{(8)}$ and Zhang \& Ren et al. ${ }^{(10)}$.

The mechanisms of patients with higher peritoneal transport had worse nutritional status were complicated. It was noticed that patients with higher peritoneal transport status had higher serum hsCRP levels and higher modified Charlson comorbidity index score, which indicated more systemic inflammation and comorbidity status. Pro-inflammatory cytokines could cause muscle wasting ${ }^{(20)}$, and inflammation might suppress appetite and induce anorexia ${ }^{(1,21)}$. In the present study, baseline higher transporters were more frequent with a decreased appetite than lower transporters both at the initiation of CAPD and follow-up period, which might indicate that a decreased appetite caused by inflammation was an important reason for the poor nutritional status at baseline. During the followup, patients with peritoneal higher transport had significantly higher estimated peritoneal glucose absorption from dialysate, and higher glucose absorption from dialysate might cause loss of appetite ${ }^{(22,23)}$. These patients also had higher incidence of peritonitis during the follow-up period. It was reported that patients with PD-related peritonitis were often with worse nutritional status, which might result from the combined effects of increased protein catabolism, increased peritoneal ALB losses and elevated acute-phase responses associated with peritoneal inflammation ${ }^{(1)}$. In another cross-sectional study, researchers also find out that both peritonitis and $4 \mathrm{~h}$ D:P cr were two independent risk factors for overall nutritional status ${ }^{(8)}$.

Keto acid has been used as therapy recommendations in PD patients with low nutrient intakes to improve the nutritional status. In the present study, patients with serum ALB less than $38 \mathrm{~g} / 1$ in both groups used keto acid for the treatment of malnutrition. Although nutritional statuses of the two groups were improved, the patients with higher peritoneal transport still had poorer nutritional status compared with that with lower peritoneal transport at the end of the study. After adjustment of keto acid supplementation and covariables such as age, sex, diabetes mellitus, RRF, Kt/V, peritonitis incidence and serum hsCRP, baseline higher peritoneal transport status was independently associated with poor nutritional status of patients at the 12 months of follow-up, which indicates that higher peritoneal transport status was an independent risk factor of malnutrition in CAPD patients.

\section{Limitations}

The present study has several noteworthy limitations. First of all, it was a single-centre study; second, we did not measure mid-arm muscle circumference in PEW assessment, but just used mBIA on measurement of muscle wasting, which probably resulted in a lower incidence of PEW than using the International Society of Renal Nutrition and Metabolism criteria. Third, the supplementation of keto acids may affect the study parameters. And yet keto acid supplementation had been adjusted both in defined binary logistic regression models and in multiple linear regressions. Finally, the study had a short follow-up duration, and longer study period is needed to observe further changes of nutritional status affected by baseline peritoneal transport status.

\section{Conclusion}

The present study demonstrated that higher transporters had higher rate of malnutrition than that of lower transporters, although nutritional status of the two groups were improved during the 12 months of CAPD. Baseline higher peritoneal transport status was an independent factor associated with worse nutritional status of CAPD patients. However, longer followup is needed to evaluate the long-term effect of baseline higher peritoneal transport status on malnutrition of CAPD patients.

\section{Acknowledgements}

We are indebted to all nephrologists and nurses in our PD centre for their excellent management of PD patients. We also thank the patients, PhD candidates and staffs involved in the prospective cohort study.

This study was supported by the Key Clinical Program of the Ministry of Health, China (2010-439), U.S. Baxter's Renal Discoveries Extramural Grant Program (EGP GRANT \#09AP012-OG), the National Key Technology R\&D Program (no. 2011BAI10B08), the National Basic Research Program of China (grant no. 2011CB504000) and the Guangzhou Committee of Science and Technology, China (grant no. 2010U1-E00831).

The authors' contributions are as follows: Y. L. contributed to the study design, data collection and drafting of the manuscript; R. H. conducted the mBIA test and SGA on patients; Q. G. and Q. Y. involved in the analysis and interpretation of the data; C. Y. and J. L. followed up the patients; X. Y. and $\mathrm{X}$. Y. involved in the study design, revision of the manuscript critically for important intellectual content and final approval of the manuscript submitted.

None of the authors has any conflict of interest to declare.

\section{References}

1. Han SH \& Han DS (2012) Nutrition in patients on peritoneal dialysis. Nat Rev Nephrol 8, 163-175.

2. Rumpsfeld M, McDonald SP \& Johnson DW (2006) Higher peritoneal transport status is associated with higher mortality and technique failure in the Australian and New Zealand peritoneal dialysis patient populations. J Am Soc Nephrol 17, 271-278.

3. Chung SH, Heimburger $\mathrm{O}$ \& Lindholm B (2008) Poor outcomes for fast transporters on PD: the rise and fall of a clinical concern. Semin Dial 21, 7-10.

4. Riella MC (2013) Nutritional evaluation of patients receiving dialysis for the management of protein-energy wasting: what is old and what is new? J Ren Nutr 23, 195-198.

5. Balafa O, Halbesma N, Struijk DG, et al. (2011) Peritoneal albumin and protein losses do not predict outcome in peritoneal dialysis patients. Clin J Am Soc Nephrol 6, 561-566. 
6. Lambie M, Chess J, Donovan KL, et al. (2013) Independent effects of systemic and peritoneal inflammation on peritoneal dialysis survival. J Am Soc Nephrol 24, 2071-2080.

7. Harty JC, Boulton H, Venning MC, et al. (1996) Is peritoneal permeability an adverse risk factor for malnutrition in CAPD patients? Miner Electrolyte Metab 22, 97-101.

8. Kang DH, Yoon KI, Choi KB, et al. (1999) Relationship of peritoneal membrane transport characteristics to the nutritional status in CAPD patients. Nephrol Dial Transplant 14, 1715-1722.

9. Szeto CC, Law MC, Wong TY, et al. (2001) Peritoneal transport status correlates with morbidity but not longitudinal change of nutritional status of continuous ambulatory peritoneal dialysis patients: a 2-year prospective study. Am J Kidney Dis 37, 329-336.

10. Zhang R \& Ren YP (2012) Protein-energy wasting and peritoneal function in elderly peritoneal dialysis patients. Clin Exp Nephrol 16, 792-798.

11. Volk ML, Hernandez JC, Lok AS, et al. (2007) Modified Charlson comorbidity index for predicting survival after liver transplantation. Liver Transpl 13, 1515-1520.

12. Lysaght MJ, Pollock CA, Hallet MD, et al. (1989) The relevance of urea kinetic modeling to CAPD. ASAIO Trans 35, 784-790

13. Twardowski ZJ, Nolph KD, Khanna R, et al. (1987) Peritoneal equilibration test. Perit Dial Bull 7, 138-147.

14. Detsky AS, McLaughlin JR, Baker JP, et al. (1987) What is subjective global assessment of nutritional status? JPEN J Parenter Enteral Nutr 11, 8-13.
15. Anonymous (1996) Adequacy of dialysis and nutrition in continuous peritoneal dialysis: association with clinical outcomes. Canada-USA (CANUSA) Peritoneal Dialysis Study Group. J Am Soc Nephrol 7, 198-207.

16. Chung SH, Han DC, Noh H, et al. (2010) Risk factors for mortality in diabetic peritoneal dialysis patients. Nephrol Dial Transplant 25, 3742-3748.

17. Barbosa-Silva MC, Barros AJ, Wang J, et al. (2005) Bioelectrical impedance analysis: population reference values for phase angle by age and sex. Am J Clin Nutr 82, 49-52.

18. Fouque D, Kalantar-Zadeh K, Kopple J, et al. (2008) A proposed nomenclature and diagnostic criteria for protein-energy wasting in acute and chronic kidney disease. Kidney Int 73, 391-398.

19. WHO Expert Consultation (2004) Appropriate body-mass index for Asian populations and its implications for policy and intervention strategies. Lancet 363, 157-163.

20. Mitch WE, Du J, Bailey JL, et al. (1999) Mechanisms causing muscle proteolysis in uremia: the influence of insulin and cytokines. Miner Electrolyte Metab 25, 216-219.

21. Mak RH, Cheung W, Cone RD, et al. (2006) Leptin and inflammation-associated cachexia in chronic kidney disease. Kidney Int 69, 794-797.

22. Chung SH, Carrero JJ \& Lindholm B (2011) Causes of poor appetite in patients on peritoneal dialysis. J Ren Nutr $\mathbf{2 1}$ $12-15$.

23. Zheng ZH, Sederholm F, Anderstam B, et al. (2001) Acute effects of peritoneal dialysis solutions on appetite in non-uremic rats. Kidney Int 60, 2392-2398. 